\title{
Rescue Model for the Bystanders' Intervention in Emergencies
}

\author{
Hang-Hyun Jo*, Woo-Sung Jung, and Hie-Tae Moon \\ Department of Physics, Korea Advanced Institute of Science and Technology, \\ Daejeon 305-701, Republic of Korea
}

\begin{abstract}
To investigate an effect of social interaction on the bystanders' intervention in emergency situations we introduce a rescue model which includes the effects of the victim's acquaintance with bystanders and those among bystanders. This model reproduces the surprising experimental result that the helping rate tends to decrease although the number of bystanders $k$ increases. The model also shows that given the coupling effect among bystanders, for a certain range of small $k$ the helping rate increases according to $k$ and that coupling effect plays both positive and negative roles in emergencies. Finally we find a broad range of coupling strength to maximize the helping rate.
\end{abstract}

Key words: Bystander effect, Social impact theory, Collective phenomena PACS: 87.23.Ge, 89.65.-s, 89.90.+n

\section{Introduction}

Recently the concepts and methods of statistical physics and nonlinear dynamics are applied to investigate social, economic and psychological phenomena $[1,2,3]$. Among the interesting subjects that have attracted physicists are the opinion dynamics $[4,5]$ including voting process [6,7] and social impact theory $[8,9,10,11,12,13]$. Social impact theory stemmed from the bystander effect that was first demonstrated in the laboratory by psychologists who studied the effect of social interaction among bystanders [14]. This effect is a marvel phenomenon where people are less likely to intervene in emergencies when others

\footnotetext{
* Corresponding author. Fax: +82-42-869-2510.

Email address: kyauou2@kaist.ac.kr (Hang-Hyun Jo).
} 
are present than when they are alone. A well known example is Kitty Genovese case [15]. She was stabbed to death at one night in 1964 by a mentally ill murderer for about 30 minutes while at least 38 witnesses were believed to have failed to help the victim. On the other hand the refutation that the popular account of the murder is mostly wrong was suggested on the website [16]. Regardless to this controversy [17] many experimental results in the laboratory and from field studies show the bystander effect obviously $[14,18]$.

Although the social impact theory originated from the bystander effect and related models have been developed, there does not exist any model describing quantitatively the bystander effect itself. To construct the model mentioned above we abstract a few important factors from the existing literatures $[14,19]$. The main dependent variables are the rates of reporting the smoke in the test room, helping endangered victims and so on. When tested alone, subjects behaved reasonably and the response rate was high. However the rate was significantly depressed when they were with other subjects. Subjects with others were unsure of what had happened or thought other people would or could do something. In another experiment subjects who were friends responded faster than those who were strangers. The subjects who had met the victim were significantly faster to report victim's distress than other subjects. And the degree of arousal that bystanders perceive is a monotonic positive function of the perceived severity and clarity of the emergency, and bystander's emotional involvement with the victim, which will be also considered by our rescue model in an abstract way.

\section{Rescue Model}

A simple rescue model is introduced for investigating the statistical feature of the effects of the victim's acquaintance with bystanders and those among bystanders in emergency situations. We focus on the relations between agents rather than agents themselves, so define a relation spin between two agents as $a_{i j}$ which is 1 if agents $i$ and $j$ have been successful in the intervention and 0 otherwise. $a_{i j}$ can be interpreted as an element of adjacency matrix of helping network. Each agent $i$ has its intervention threshold $c_{i}$ over which that agent can try to intervene in an emergency situation.

At each time step an accident happens which consists of the degree of accident represented as a random number $q_{v}$ uniformly drawn from $[0,1]$, a randomly selected victim $v$, the number of bystanders $k$ confined to $[1, N-1]$, and a set of randomly selected $k$ bystanders $N_{v}$ from a population. Then the update 
rule is as following:

$$
a_{v i}(t+1)=\theta\left(q_{v}+\alpha a_{v i}(t)+\beta \sum_{j \in N_{v}, j \neq i}\left(2 a_{i j}(t)-1\right)-c_{i}\right)
$$

where $\theta\left(x_{i}\right)$ is a heaviside step function. Only one bystander $i \in N_{v}$ with the largest value $x_{i}$ can intervene per accident. If we assume that the response speed of bystander $i$ is exponential in $x_{i}$, the selection of the bystander with the largest $x_{i}$ is justified. Additionally, once one bystander intervenes, the pressures on the others will disappear. If $x_{i} \geq 0$, the rescue succeeds and then the victim $v$ and that bystander $i$ gain acquaintances if they have not been related to each other. In case of $x_{i}<0$ the rescue fails and then their acquaintance is cut if existed. $\alpha$ represents the degree of victim's acquaintance with bystander, so can be called an acquaintance strength. The third term of $\theta$ function is related to the acquaintances among bystanders, for each relation $2 a_{i j}-1$ gives 1 if two bystanders know each other or -1 otherwise. There does not exist any neutral relation here. $\beta$ is used to tune the strength of coupling so can be called a coupling strength. As an order parameter we adopt the average helping rate:

$$
\langle a\rangle(t)=\frac{2}{N(N-1)} \sum_{i<j} a_{i j}(t)
$$

which can be also interpreted as a social temperature or an average linkage of the helping network. We fix $c_{i} \equiv c=0.25$ for all $i$ according to the experimental result [14] that $70 \sim 75 \%$ of alone subjects intervened and $c$ does not change through this paper, which means we consider a population composed of homogeneous and non-adaptive agents. And for the most cases $\alpha$ is also fixed as 0.1 . The main control parameter is the number of bystanders $k$. Finally, the initial conditions are $a_{i j}=0$ for all pairs.

\subsection{Case with $\beta=0$}

At first let us consider only the effect of victim's acquaintance with bystanders and ignore those among bystanders. Generally an equation for the average helping rate can be written as

$$
\frac{d a_{k}(t)}{d t}=W_{0 \rightarrow 1}-W_{1 \rightarrow 0}
$$

where $a_{k}(t)$ denote $\langle a\rangle(t)$ for a fixed $k$. Given the values of parameters as above, when $0 \leq q_{v}<c-\alpha$, the rescue fails independent of $a_{v i}(t)$ so one of acquaintances, if exists, should be cut. When $c-\alpha \leq q_{v}<c, a_{v i}(t+1)=a_{v i}(t)$ so this interval of $q_{v}$ does not contribute to the equation. When $c \leq q_{v} \leq 1$ the 
rescue succeeds independent of $a_{v i}(t)$ so a new acquaintance is formed only if there is no acquaintance between them.

$$
\begin{aligned}
& W_{0 \rightarrow 1}=(1-c)\left(1-a_{k}(t)\right)^{k} \\
& W_{1 \rightarrow 0}=(c-\alpha)\left(1-\left(1-a_{k}(t)\right)^{k}\right)
\end{aligned}
$$

The stationary solution is obtained easily.

$$
a_{k}=1-\left(\frac{c-\alpha}{1-\alpha}\right)^{1 / k}
$$

The solution means that although the coupling strength is not considered $(\beta=0)$, the helping rate depends on the number of bystanders $k$. As increasing $k$ the probability that the victim does not know any bystanders (contributes to $W_{0 \rightarrow 1}$ ) decreases rapidly and the complementary probability (contributes to $\left.W_{1 \rightarrow 0}\right)$ increases. This is why the helping rate decays. In this case $\alpha$ affects not the decaying behavior but the decaying speed only when $\alpha<c$. If $\alpha$ becomes larger than $c, W_{1 \rightarrow 0}=0$ so $a_{k}=1$ independent of $k$.

The numerical simulations are shown in Fig. 1 and reproduce the experimental results that people who live in larger cities are less likely to help the strangers [18]. Compared to Ref. [18] $k$ corresponds to the size of city or town.

\subsection{Case with $\beta>0$ and $k=2$}

If the coupling among bystanders is taken into account and $k=2$, the interval $[0,1]$ of $q_{v}$ is divided into five subintervals by four points $c-\alpha-\beta, c-\alpha+\beta$, $c-\beta$, and $c+\beta$. Whether $c-\alpha+\beta$ is smaller or larger than $c-\beta$ does not affect the result. For each subinterval the transition rate is calculated.

$$
\begin{aligned}
& W_{0 \rightarrow 1}=(1-c-\beta)\left(1-a_{2}\right)^{3}+(1-c+\beta) a_{2}\left(1-a_{2}\right)^{2} \\
& W_{1 \rightarrow 0}=(c-\alpha+\beta) a_{2}\left(1-a_{2}\right)\left(2-a_{2}\right)+(c-\alpha-\beta) a_{2}^{2}\left(2-a_{2}\right)
\end{aligned}
$$

The second term in R.H.S. of $W_{1 \rightarrow 0}$ vanishes when $c-\alpha-\beta<0$, so we get the solutions for both cases.

$$
a_{2}= \begin{cases}\frac{1-\alpha-\beta-\sqrt{\alpha^{2}+\beta^{2}+\alpha \beta-\alpha-\beta+c(1-\alpha)}}{1-\alpha} & \text { if } c-\alpha-\beta \geq 0 \\ \frac{1+c-2 \alpha-\beta-\sqrt{5 c^{2}+4 \alpha^{2}-3 \beta^{2}-8 \alpha c-2 \beta c-2 c+2 \beta+1}}{2(c-\alpha-\beta)} & \text { if } c-\alpha-\beta<0\end{cases}
$$

The numerical simulations in Fig. 2 support the analytic solution. One can find a maximum value of $a_{2}$ when $c-\alpha-\beta=0$, which means that there exists 


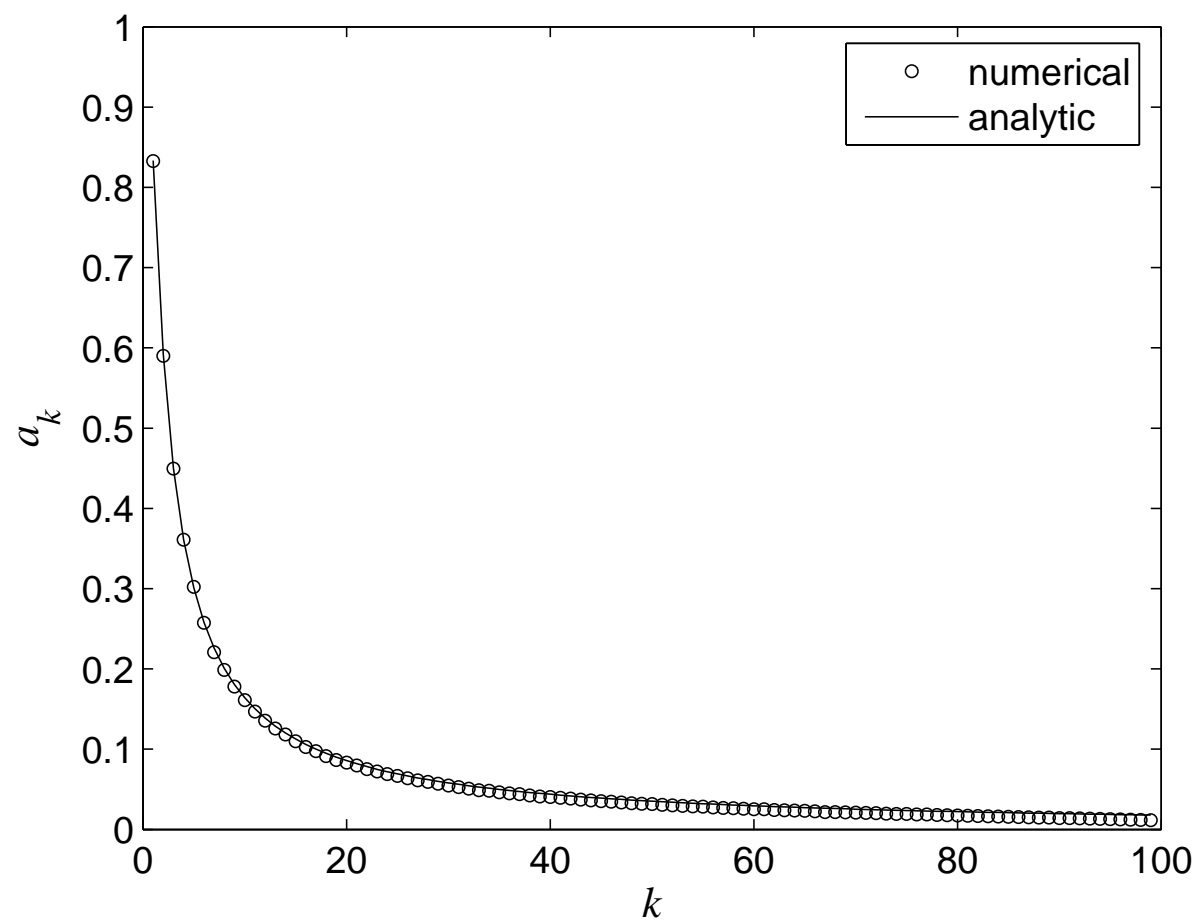

Fig. 1. The analytic solution and numerical simulations of the case with $\alpha>0$ and $\beta=0$. Here $N=100, c=0.25$ and $\alpha=0.1$.

an optimal coupling strength $\beta_{\text {opt }}=c-\alpha$ to maximize the helping rate when given $c$ and $\alpha$.

\subsection{General case with $\beta>0$}

For a general $k$, the interval $[0,1]$ of $q_{v}$ is divided by the following points: $c-\alpha-(k-1) \beta, \cdots, c-\alpha+(k-1) \beta, c-(k-1) \beta, \cdots, c+(k-1) \beta$. For each subinterval the transition rate can be calculated.

$$
\begin{aligned}
& W_{0 \rightarrow 1}=\left(1-a_{k}\right)^{k}\left(1-c+(k-1) \beta-2 \beta F\left(a_{k}, k\right)\right) \\
& W_{1 \rightarrow 0}=\left(1-\left(1-a_{k}\right)^{k}\right)\left(c-\alpha-(k-1) \beta+2 \beta F\left(a_{k}, k\right)\right)
\end{aligned}
$$

where

$$
F\left(a_{k}, k\right)=\sum_{n=0}^{k-2}(k-1-n)\left(\begin{array}{c}
\frac{1}{2} k(k-1) \\
n
\end{array}\right) a_{k}^{n}\left(1-a_{k}\right)^{\frac{1}{2} k(k-1)-n}
$$

Consequently to get a stationary solution we must solve the following equation.

$$
(1-\alpha)\left(1-a_{k}\right)^{k}=c-\alpha-(k-1) \beta+2 \beta F\left(a_{k}, k\right)
$$




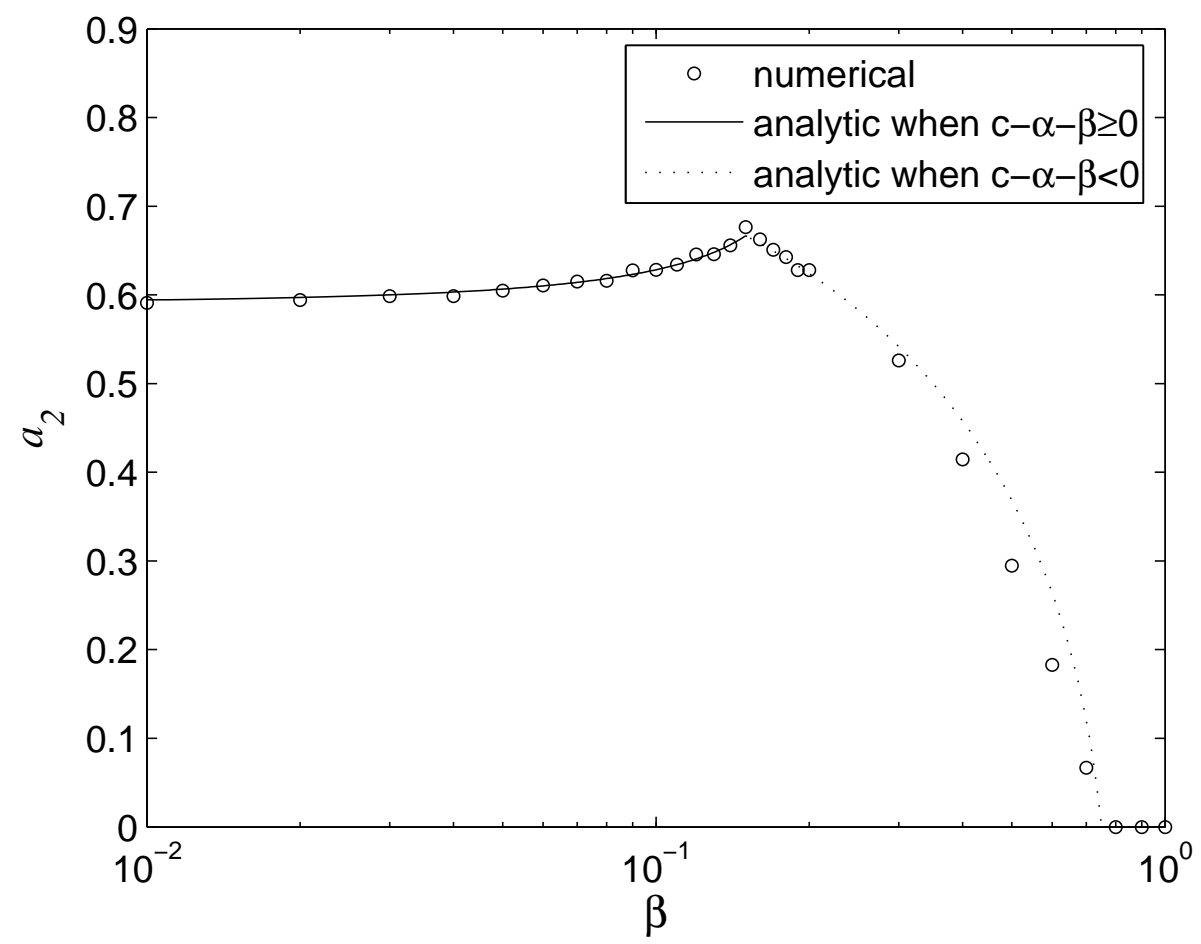

Fig. 2. The analytic solution and numerical simulations of the case with $\alpha, \beta>0$ and $k=2 . a_{2}$ has a maximum value when $c-\alpha-\beta=0$. The other parameter values are the same as Fig. 1.

Since $F\left(a_{k}, k\right)$ is the $\frac{1}{2} k(k-1)$ th order polynomial, the order of above equation is $\max \left(\frac{1}{2} k(k-1), k\right)$ and difficult to solve exactly for all $k$. Therefore, as an approximation we consider only the most left, the second and the most right subintervals.

$$
\begin{aligned}
& W_{0 \rightarrow 1}=(1-c-(k-1) \beta)\left(1-a_{k}\right)^{k} \\
& W_{1 \rightarrow 0}=(c-\alpha-(k-3) \beta)\left(1-\left(1-a_{k}\right)^{k}\right)
\end{aligned}
$$

The approximate stationary solution is

$$
a_{k}=1-\left(\frac{c-\alpha-(k-3) \beta}{1-\alpha-2(k-2) \beta}\right)^{1 / k} \text { when } k \leq k_{1} \equiv \frac{c-\alpha}{\beta}+3
$$

Figure 3 shows the numerical simulations supporting the approximation except for $k \approx k_{1}$. The helping rate undergoes a trough for $k$ less than $k_{1}$, which is also partly observed in the experiment results [18], and then decreases monotonically as for the case without a coupling effect $(\beta=0)$. For $k \geq k_{2}$ the helping rate shows a sudden drop to 0 . To understand this behavior intuitively two factors should be considered. On the one hand as for the case without a coupling effect, the increasing number of bystanders in- 


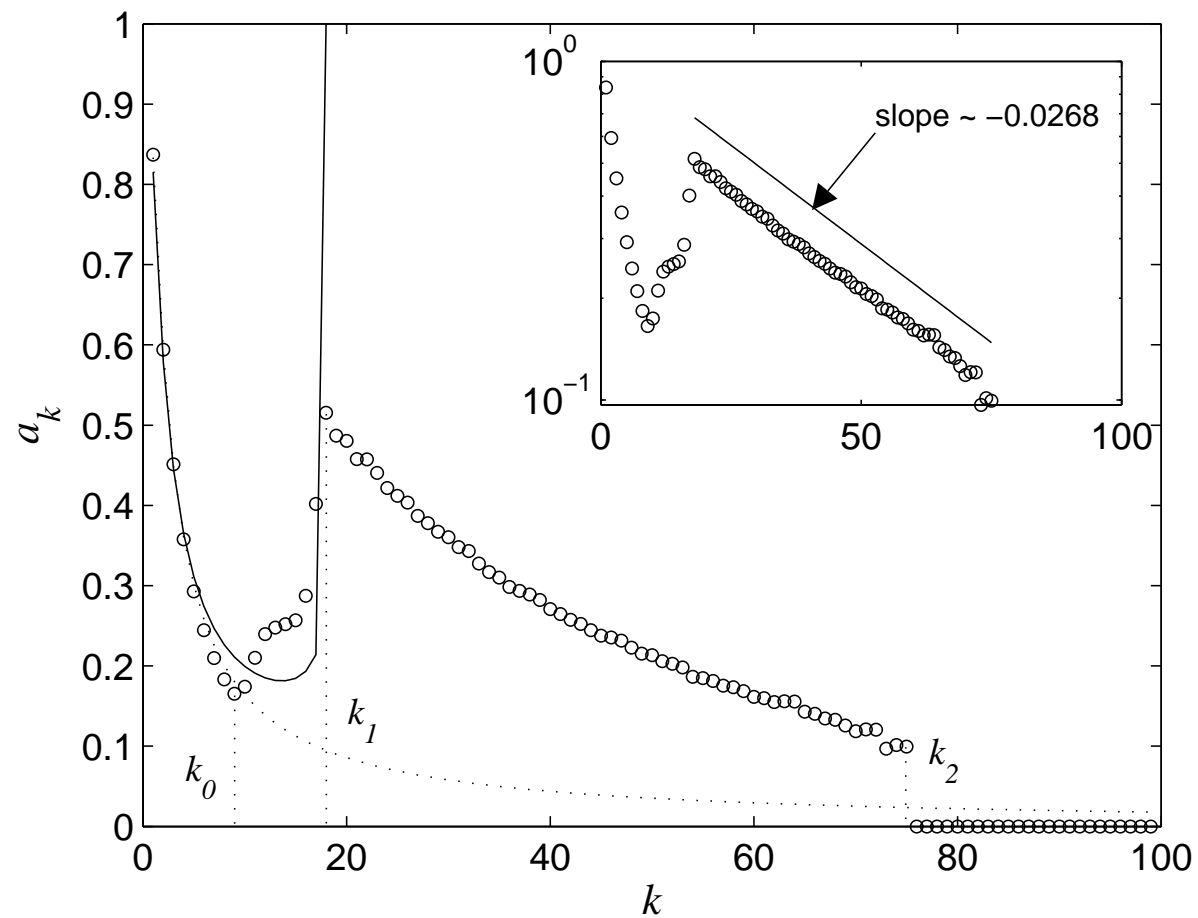

Fig. 3. The analytic solution and numerical simulations of the case with $\beta=0.01$. Circles represent the numerical results and solid line and dotted line do the approximate solution for $\beta=0.01$ and for $\beta=0$ for comparison respectively. Inset graph shows the exponentially decaying behavior of the same data.

creases the probability of cutting acquaintance too. On the other hand the most left subinterval $[0, c-\alpha-(k-1) \beta)$, which most contributes to $W_{1 \rightarrow 0}$, decreases as increasing $k$. In this paper $c$ is fixed as a rather small value so that a change of the most right subinterval rarely affects $W_{0 \rightarrow 1}$. In conclusion, when $k<k_{0}$ the former factor dominates the latter so that the helping rate decreases. When $k_{0} \leq k<k_{1}$ the increasing $k$ minimizes the inhibiting effect by other bystanders to make the helping rate increase. Once $k$ passes $k_{1}$, the decreasing subinterval vanishes and only the former factor works.

Coupling effect among bystanders plays both positive and negative roles in emergencies. When $k<k_{0}$, the helping rate is rarely affected by the coupling. In case of $k_{0} \leq k \leq k_{2}$, the coupling helps the helping rate obviously. Especially when $k_{1} \leq k \leq k_{2}, a_{k}$ decays exponentially with slope of -0.0268 in the semi-log plot. Once $k$ passes $k_{2}, a_{k}$ turns to 0 because of too many other inhibiting bystanders. The value of $k_{2}$ can be obtained by a simple mean field 


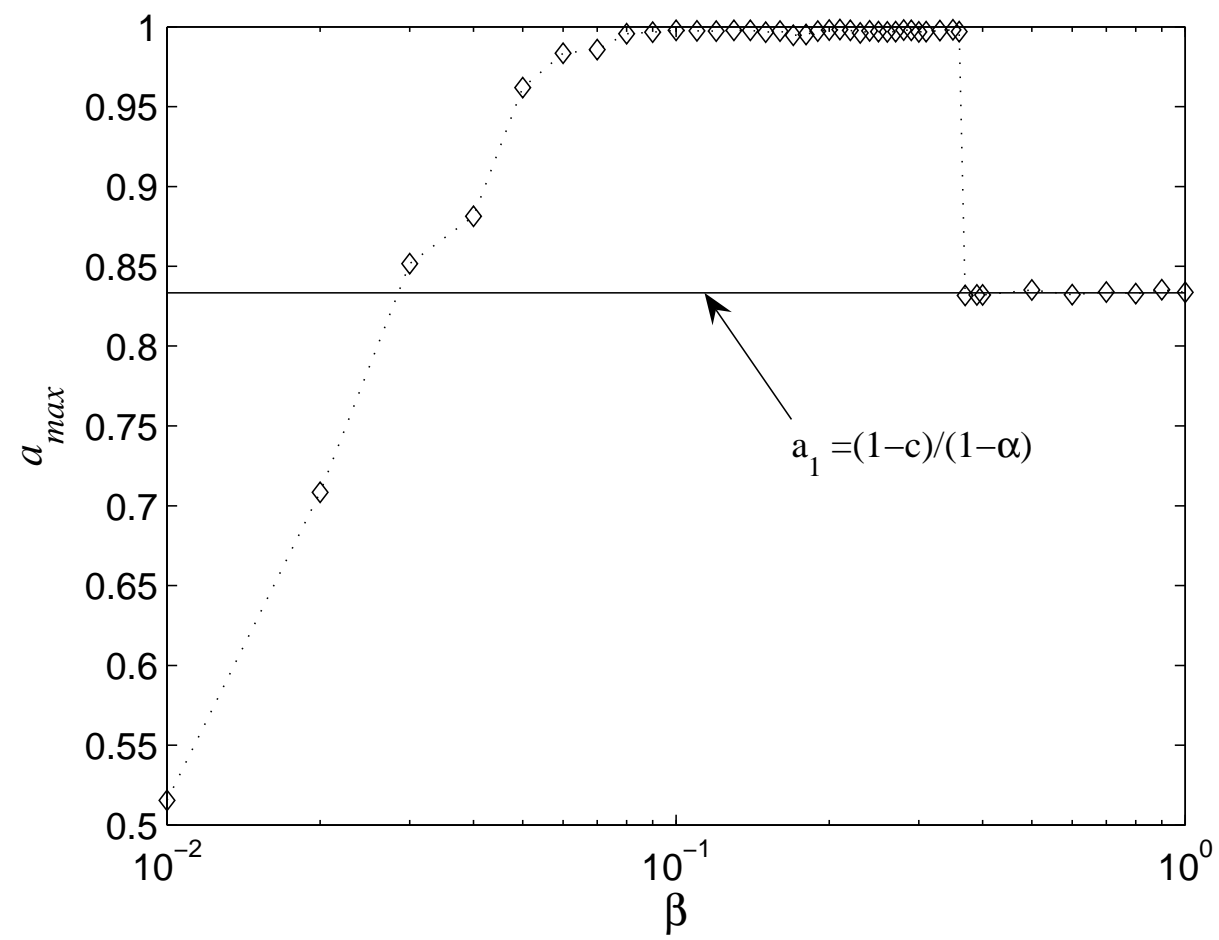

Fig. 4. The maximum value $a_{\max }$ as a function of $\beta$. There exists a broad range of $\beta$ to maximize the helping rate.

approximation and by using the following:

$$
\left\langle\theta\left(x-x_{0}\right)\right\rangle_{x}= \begin{cases}1 & \text { if } x_{0} \leq 0 \\ 1-x_{0} & \text { if } 0<x_{0} \leq 1 \\ 0 & \text { if } x_{0}>1\end{cases}
$$

$\langle\cdot\rangle_{x}$ means an average over $x \in[0,1]$. From Equation (1), if $c-\alpha a_{k}-\beta(k-$ 1) $\left(2 a_{k}-1\right)>1$, then $a_{k}=0$, therefore the condition for $a_{k}=0$ is $k>k_{2} \equiv$ $\frac{1-c}{\beta}+1$.

Under what conditions is the helping rate maximized? For each $(\alpha, \beta)$ we can find the maximum value of $a_{k}$, denoted by $a_{\max }$. It is sufficient to compare $a_{1}$ to $a_{k_{1}}$, where $k_{1}$ is defined by Eq. 16. $a_{1}$ is $\frac{1-c}{1-\alpha}$ independent of $\beta$ while $a_{k_{1}}$ is smaller than $a_{1}$ for small $\beta$ and exceeds $a_{1}$ for some range of $\beta$ and then vanishes, in fact $k_{1}=1$ for larger $\beta$ in Fig. 4 . Especially $a_{\max }$ approaches to 1 when $0.08 \leq \beta \leq 0.35$. There exists a broad range of $\beta$ for which the helping network is almost fully connected. 


\section{Conclusions}

We introduced a simple rescue model to investigate the effects of victim's acquaintance with bystanders (acquaintance strength $\alpha$ ) and those among bystanders (coupling strength $\beta$ ) for the bystanders' intervention in emergency situations. When $\beta=0$, as the increasing number of bystanders $k$ the helping rate decreases, where the speed depends on $\alpha$. For the case of $\beta>0$ and $k=2$, there exists an optimal coupling strength $\beta=c-\alpha$ for the maximum helping rate. Coupling strength plays both positive and negative roles in emergencies. For $k_{0} \leq k<k_{1}$, since the coupling among bystanders minimizes the inhibiting effect, the helping rate increases according to $k$. And then the helping rate decays monotonically as for the case without a coupling effect. Once $k$ passes

$k_{2}$ too many bystanders inhibit the helping. There exists a rather broad range of $\beta$ where almost all the trials to intervene in emergencies are successful. In this case it is not necessary to fine-tune the coupling strength to get the highest helping rate.

\section{References}

[1] W. Weidlich, Phys. Rep. 204 (1991) 1-163.

[2] R. N. Mantegna and H. E. Stanley, An Introduction to Econophysics, Cambridge University Press, 2000.

[3] R. R. Vallacher and A. Nowak (Eds.), Dynamical Systems in Social Psychology, Academic Press, 1994.

[4] D. Stauffer, arXiv:physics/0503115.

[5] K. Sznajd-Weron and J. Sznajd, Int. J. Mod. Phys. C 11 (2000) 1157-1165; K. Sznajd-Weron, Phys. Rev. E 66 (2002) 046131; K. Sznajd-Weron, Phys. Rev. E 70 (2004) 037104.

[6] S. G. Alves, N. M. Oliveira Neto, and M. L. Martins, Physica A 316 (2002) 601-604.

[7] A. T. Bernardes, D. Stauffer, and J. Kertész, Eur. Phys. J. B 25 (2002) 123-127.

[8] B. Latané, Am. Psychologist 36 (1981) 343-356.

[9] A. Nowak, J. Szamrej and B. Latané, Psychol. Rev. 97 (1990) 362-376.

[10] M. Lewenstein, A. Nowak and B. Latané, Phys. Rev. A 45 (1992) 763-776.

[11] G. A. Kohring, J. Phys. I France 6 (1996) 301-308.

[12] D. Plewczyński, Physica A 261 (1998) 608-617. 
[13] J. A. Hołyst, K. Kacperski and F. Schweitzer, Physica A 285 (2000) 199-210; J. A. Hołyst and K. Kacperski, Annual Reviews of Computational Physics IX, World Scientific, 2001, p. 253-273.

[14] B. Latané and J. M. Darley, American Scientist 57 (1969) 244-268.

[15] http://en.wikipedia.org/wiki/Kitty_Genovese

[16] J. De May Jr., http://www.oldkewgardens.com/ss-nytimes-3.html

[17] J. Rasenberger, The New York Times, February 8, 2004.

[18] P. R. Amato, J. Person. Soc. Psychol. 45 (1983) 571-586.

[19] J. A. Piliavin, J. F. Dovidio, S. L. Gaertner, and R. D. Clark III, Responsive bystanders: The process of intervention. In V. J. Derlega and J. Grzelak (Eds.), Cooperation and helping behavior: Theories and research, Academic Press, 1982. 